\title{
Editorial: A Celebration of the Ties That Bind Us: Connections between Actuarial Science and Mathematical Finance
}

\begin{abstract}
Albert Cohen
Department of Mathematics, Michigan State University, East Lansing, MI 48823, USA; albert@math.msu.edu

Received: 5 January 2018; Accepted: 9 January 2018; Published: 15 January 2018

Abstract: In the nearly thirty years since Hans Buhlmann (Buhlmann (1987)) set out the notion of the Actuary of the Third Kind, the connection between Actuarial Science (AS) and Mathematical Finance (MF) has been continually reinforced. As siblings in the family of Risk Management techniques, practitioners in both fields have learned a great deal from each other. The collection of articles in this volume are contributed by scholars who are not only experts in areas of AS and MF, but also those who present diverse perspectives from both industry and academia. Topics from multiple areas, such as Stochastic Modeling, Credit Risk, Monte Carlo Simulation, and Pension Valuation, among others, that were maybe thought to be the domain of one type of risk manager are shown time and again to have deep value to other areas of risk management as well. The articles in this collection, in my opinion, contribute techniques, ideas, and overviews of tools that specialists in both AS and MF will find useful and interesting to implement in their work. It is also my hope that this collection will inspire future collaboration between those who seek an interdisciplinary approach to risk management.
\end{abstract}

Keywords: actuarial science; mathematical finance; risk management

\section{Background and Motivation}

The construction of this special issue began in 2016, and continued through 2017, while teaching at Michigan State University (MSU) as well as visiting colleagues from the Pacific Northwest to New York City to the Deep South. I had known for some time that I wanted to design an issue that would present cutting edge research in both actuarial science and financial mathematics. In truth, I had been inspired nearly a decade earlier by this strong connection between both fields. As a Ph.D. student at Carnegie Mellon, I had come across Hans Gerber's paper (Gerber (1977)) on the optimal cancellation of policies. At the time, I had begun working on a problem in optimal prediction and was looking for inspiration in solving the problem at hand. When I came across Gerber's paper, I had no previous exposure to actuarial science, but I immediately recognized the power and clarity of his exposition. I was hooked! I did eventually solve the problem in optimal prediction (Cohen (2010)) a few years later, but not without using ideas from Gerber's model of liabilities as an application of optimal prediction to a new kind of risk measure. And so began my education in the overlap of the fields of insurance and financial economics.

\section{Overview}

This collection you now hold in your hand is crafted in the spirit of Gerber's paper, in that advanced mathematical techniques and risk management go hand in hand to present managers with better information for decision making.

For example, new ideas such as those presented in Peter Carr's work (Carr (2017)) on Bounded Brownian Motion naturally find their place in the modeling of financial instruments, but are perhaps 
also suitable for insurance models that require such bounded processes. Stephen Mildenhall's paper (Mildenhall (2017)) on Actuarial Geometry combines tools from the classical study of shapes with Levy processes to provide an innovative way to study risks from an insurance perspective. Robert J. Rietz and his group (Rietz et al. (2017)) study the effect of gainsharing (via simulation) on selecting discount rates for defined benefit plans. Gareth W. Peters, Rodrigo S. Targino and Mario V. Wuthrich (Peters et al. (2017)) provide a novel Monte Carlo method to calculate (coherent) capital allocations for a general insurance company. Carolyn W. Chang and Jack S. K. Chang (Chang and Chang (2017)) utilize an approach that integrates commonly used tools from actuarial science and mathematical finance to price a default-risky catastrophe reinsurance contract. Daoping Yu and Vytaras Brazauskas (Yu and Brazauskas (2017)) study the impact of model uncertainty on value-at-risk (VaR) estimators. In her paper on predicting prices for high profile tech stocks, Nguyet Nguyen (Nguyen (2017)) applies the Hidden Markov Model (HMM) to forecast stock prices and develop an HMM-based trading strategy. Michael R. Metel, Traian A. Pirvu and Julian Wong (Metel et al. (2017)) investigate the Omega Measure and it's use for assessing portfolio performance, as well as similarities and differences with the Sharpe Ratio when determining the optimal portfolio for different return classes. Finally, Nick Costanzino and I (Cohen and Costanzino (2017)) look at incorporating stochastic recovery into the Black-Cox model of bond pricing, with application to credit default swaps.

\section{Conclusions}

This collection of articles fits into the greater narrative of collaboration between researchers and practitioners, driven by new and innovative ideas and products in finance and insurance. The connection between AS and MF, for actuaries of the third kind (Buhlmann (1987)) and others who use these tools, will only strengthen with time as the complexity of financial and insurance instruments increases.

Acknowledgments: There are so many colleagues in both finance and actuarial science that I would like to thank for their friendship and guidance. In Actuarial Science, I would like to thank my dear friend Emil Valdez (UConn) for his leadership and dedication to research in the field as well as the education of the next generation of actuaries. I would also like to thank Virginia Young and Kristen Moore at the University of Michigan, and in the East Lansing/MSU community, thank you to all of my students (past and present), Ron and Mary Simon, Mark Wenger, Kevin Clinton, Jacob Geyer, Wellington Ow, Gabor Franciscs, Darren Mason, and Robert J. Rietz for their continued support and mentorship. Thank You to Keith Promislow for bringing me to MSU, and to Yang Wang, now at the Hong Kong University of Science and Technology, for hiring me to help build the major in actuarial science at Michigan State. For his wonderful paper on Actuarial Geometry, I would like to acknowledge Stephen Mildenhall. Stephen's paper is not only an achievement in terms of research, but is also a valuable resource for young researchers in insurance who wish to learn about stochastic processes and geometry from an actuarial perspective. In Finance, thank you to Steven Shreve and Dmitry Kramkov for giving me a solid education in mathematical finance while I was a graduate student at Carnegie Mellon. Also, thank you to all in the field who have extended the hand of friendship and brought me into your community. These include, among many scholars: Peter Carr (NYU-Tandon), Nguyet Nguyen (Youngstown State), Harvey Stein, Sean Hilden, Shlomo Levental (MSU), and Mark Schroder (MSU). I would like to especially thank my friend and research partner Nick Costanzino for his insight and introduction to so many neat areas of cutting edge finance and mathematics. I truly would not be in the position I am now without his continued support. Finally, I would like to thank my family for their support and patience over the last couple years while this special collection of articles took shape. I spent many late nights working on this project while they patiently waited at home for me to come back and spend time with them. To my beautiful wife Jessica and our son Eli, I love you! In closing, I would like to dedicate my work in editing this collection to the memory of my Godfather, Asher Cohen.

Conflicts of Interest: The author declare no conflict of interest.

\section{References}

Bühlmann, Hans. 1987. Actuaries of the Third Kind (editorial). ASTIN Bulletin 17: 137-38.

Carr, Peter. 2017. Bounded Brownian Motion. Risks 5: 61. doi:10.3390/risks5040061.

Chang, Carolyn, and Jack Chang. 2017. An Integrated Approach to Pricing Catastrophe Reinsurance. Risks 5: 51. doi:10.3390/risks5030051. 
Cohen, Albert, and Nick Costanzino. 2017. Bond and CDS Pricing via the Stochastic Recovery Black-Cox Model. Risks 5: 26. doi:10.3390/risks5020026.

Cohen, Albert. 2010. Examples of optimal prediction in the infinite horizon case. Statistics \& Probability Letters 80: 950-57.

Gerber, Hans U. 1977. On optimal cancellation of policies. ASTIN Bulletin: The Journal of the IAA 9: 125-38.

Metel, Michael R., Traian A. Pirvu, and Julian Wong. 2017. Risk Management under Omega Measure. Risks 5: 27. doi:10.3390/risks5020027.

Mildenhall, Stephen. 2017. Actuarial Geometry. Risks 5: 31. doi:10.3390/risks5020031.

Nguyen, Nguyet. 2017. An Analysis and Implementation of the Hidden Markov Model to Technology Stock Prediction. Risks 5: 62. doi:10.3390/risks5040062.

Peters, Gareth W., Rodrigo S. Targino, and Mario V. 2017. Bayesian Modelling, Monte Carlo Sampling and Capital Allocation of Insurance Risks 5: 53. doi:10.3390/risks5040053.

Rietz, Robert, Evan Cronick, Shelby Mathers, and Matt Pollie. 2017. Effects of Gainsharing Provisions on the Selection of a Discount Rate for a Defined Benefit Pension Plan. Risks 5: 32. doi:10.3390/risks5020032.

Yu, Daoping, and Vytaras Brazauskas. 2017. Model Uncertainty in Operational Risk Modeling Due to Data Truncation: A Single Risk Case. Risks 5: 49. doi:10.3390/risks5030049.

(C) 2018 by the author. Licensee MDPI, Basel, Switzerland. This article is an open access article distributed under the terms and conditions of the Creative Commons Attribution (CC BY) license (http://creativecommons.org/licenses/by/4.0/). 